Fourth Meeting, Friday, 9th February, 1900.

R. F. Moirhead, Esq., M.A., B.Sc., President, in the Chair.

\title{
Remark on Dr Peddie's Proof of the Potential Theorems regarding Uniform Spherical Shells.
}

By R. F. Muirhead, M.A., B.Sc.

On reading Dr Peddie's paper, the following modification of the proof, which avoids summation, occurred to me :-

If in Figure 7 we take a point $S$ on the circle $B Q D$ such that $\mathrm{PQ}+\mathrm{PS}=2 a$, where $a$ is the radius, and a corresponding point $\mathrm{S}^{\prime}$ such that $\mathrm{PQ}^{\prime}+\mathrm{PS}^{\prime}=2 a$, then it is clear by $\mathrm{Dr}$ Peddie's construction that the potential at $\mathbf{P}$ due to the zone of the spherical surface lying between planes through $Q$ and $Q^{\prime}$ perpendicular to $B D$ is given by $2 \pi \sigma\left(P Q^{\prime}-P Q\right) . a / C P$, and is therefore equal to that due to the corresponding zone between $S$ and $\mathrm{S}^{\prime}$, since

$$
P^{\prime}-P Q=P S-P S^{\prime} \text {. }
$$

The potentials at $\mathrm{P}$ due to these zones being respectively $\frac{m}{\mathrm{PQ}}$ and $\frac{m^{\prime}}{\mathrm{PS}}$, where $m$ and $m^{\prime}$ are the masses of these zones, and these potentials being equal, their sun is

$$
\frac{2\left(m+m^{\prime}\right)}{\mathrm{PQ}+\mathrm{PS}}=\frac{2\left(m+m^{\prime}\right)}{2 \boldsymbol{a}}=\frac{m+m^{\prime}}{\boldsymbol{a}} .
$$

Thus the potential due to these parts of the surface is the same as if they were placed at distance $a$ from P. But since the whole spherical surface is divisible into such corresponding pairs of zones, the potential at $P$ due to the whole surface is the same as if its mass were all at distance $a$ from $P$, i.e., the same as when $P$ is at $C$.

The foregoing applies to the case when $P$ is an internal point, but the modification for the case of $\mathbf{P}$ external is easily made.

\section{A general mechanical description of the Conic Sections.}

By Alex. Morrison, M.A., B.Sc. 